\title{
Schreiben: Kognition und Körperlichkeit in einem
}

\section{Thomas Lindauer und Afra Sturm}

"Wie den Seefahrern der Hafen willkommen ist, so auch dem Schreiber die

letzte Zeile. Wer das Schreiben nicht kennt, meint, für ihn sei das keine Arbeit, denn während drei Finger schreiben, arbeitet der ganze Körper." (7./8. Jh. Verona, Biblioteca capitolare, Steinmann, 2013, S. 68)

In der deutschsprachigen Schreibforschung und-didaktik spielt der Ansatz der konzeptionellen Schriftichkeit eine zentrale Rolle. So relevant dieser Ansatz auch ist, so wird mit ihm doch die materielle Bedingtheit des Schreibens ausgeblendet. In der neueren und vor allem auch empirischen Schreibforschung zeigt sich, dass der Erwerb der basalen Schreibfertigkeiten, der insbesondere die "Körperlichkeit» der Textproduktion einschliesst, eine wichtige Voraussetzung darstellt, um erweiterte Schreibkompetenzen in einem ausreichenden Mass zu erwerben. In diesem Beitrag wird einerseits aufgezeigt, was unter basalen Schreibfertigkeiten bzw. unter "Schriftproduktion" zu verstehen ist und welche Rolle sie genau für den Schreiberwerb und damit auch für die Deutschdidaktik spielt; andererseits soll mit Bezug auf das SNF-Projekt "Transformation schulischen Wissens seit 1830" die Bedeutung der Materialität (von der Breitfeder über die Füllfeder hin zu zum Softpen u.a.) für die Didaktik der Körperhaltung beim Schreiben im historischen Wandel dargestellt werden.

\section{Einleitung}

Schreiben verstanden als Textproduktion ist eine komplexe und kognitiv wie auch physisch anspruchsvolle Tätigkeit: Beim Verfassen von Texten werden verschiedene kognitive Prozesse gebündelt und miteinander koordiniert. Diese wiederum müssen mit (fein-)motorischen Aktivitäten abgestimmt werden, da die Textproduktion auch «Schriftproduktion» beinhaltet. Nicht zuletzt konkurrieren die motorischen mit den kognitiven Aktivitäten um die begrenzten Ressourcen des Arbeitsgedächtnisses. Dies gilt nicht nur für Schreibnovizen und 
-novizinnen, sondern auch für ältere Schüler und Schülerinnen sowie Erwachsene - wenn auch bei Schreibnovizen und -novizinnen in anderem Masse (Bourdin \& Fayol, 2002). Schreiben sollte jedoch nicht auf die kognitive und motorische Dimension begrenzt werden (Prior, 2008): Ein Grossteil der Schreibformen weist auch eine soziale Dimension auf und wird daher als ein Problemlöseverfahren verstanden, das in eine soziale bzw. kommunikative Situation eingebettet ist (Sturm \& Weder, 2011).

Diese Sichtweise auf Schreiben lässt sich in einer Forschungstradition verorten, die spätestens mit Hayes und Flower (1980) einsetzt: Zum einen wurde auf dieser Basis mit Self regulated strategy development (SRSD) ein Ansatz entwickelt, der eine stark kognitivistische Orientierung aufweist, den ursprünglich behavioristischen Zugang aber nicht ganz aufgibt (Graham \& Harris, 2009). Zum anderen etablierte sich - auch im deutschen Sprachraum - eine prozessorientierte Schreibforschung und Schreibdidaktik, auf deren Basis die Lernenden in die Prozesse Planen, Formulieren und Überarbeiten rekursiv engagiert werden (Becker-Mrotzek \& Böttcher, 2012; Fix, 2008). Schliesslich wird Schreiben auch als Schreibhandlung verstanden, die zusätzlich kulturelle sowie funktionale Aspekte aufweist (Feilke, 2011).

In all diesen Ansätzen spielt die motorisch-produktive, materialisierte Schreib- bzw. Schriftproduktion kaum eine Rolle - dies im Gegensatz zu den Anfängen der Schreibdidaktik im 19. Jahrhundert, welche Schreiben primär als Schriftfertigkeit und als (Re-)Produktion von Textmustern verstand. Schreiben als (fein-)motorische Tätigkeit findet nach einer langen Tradition bis in die 1960er-Jahre hinein erst wieder in neueren Schreibprozessmodellen Eingang. So unterscheidet denn auch Hayes (1996, 2012) zwischen Formulieren und Transkribieren: Formulieren meint das Überführen von Ideen in sprachliche Repräsentation, während Transkribieren das Überführen von sprachlicher Repräsentation in Schrift bezeichnet. Hayes (2012, S. 24) betont, dass beide Aktivitäten eine enge Verbindung eingehen und die Schriftproduktion den Schreibprozess bspw. spürbar verlangsamen kann.

Aus einer didaktischen Perspektive bemerkenswert ist der Umstand, dass in prozessorientierten Ansätzen Transkribieren dennoch weitgehend ausgeblendet wird. Exemplarisch dafür seien Abraham und Kupfer-Schreiner (2007) genannt, die die Grundlagen eines prozessorientierten Schreibunterrichts für die Klassen 1-4 formulieren: Sie führen zwar motorische (Produktions-)Prozesse als Element in ihrem Schreibprozessmodell an, modellieren dies aber weder didaktisch-konzeptionell noch methodisch-konkret. Entsprechend werden motorische Prozesse und die darauf abzustimmende Körperhaltung beim Schreiben in ihren Unterrichtsbeispielen, die für gute Schreibaufgaben stehen, nicht thematisiert.

Dass eine in der Kognition angelegte Schnittstelle vom Formulieren zum Transkribieren und damit auch zur Grafomotorik in seiner fürs Schreibenlernen relevanten materiellen Konkretheit vage bleibt, mitunter die mediale Bedingtheit des Schreibens und Schreibenlernens ausgeblendet wird, wird insbesondere von 
Wrobel (2010) kritisch diskutiert. Pointiert leitet er seinen Beitrag wie folgt ein: Was macht einen Text aus? Ist es eher sein gedankliches Konzept oder die spezifische Umsetzumg im Medium der Schrift? Wozu braucht ein Schreiber seine Hände? Braucht er sie überhaupt? (Wrobel, 2010, S. 27)

Im Folgenden möchten wir einen Denkrahmen skizzieren, der Schreiben zusätzlich zur kognitiv-strukturellen und sozialen Dimension auch in seiner körperlichen Dimension in den Blick bringt. Dazu gehen wir auch der Frage nach, wie die materielle Bedingtheit der Schreibwerkzeuge spezifische didaktische Ansprüche an das Vermitteln von Schreibfähigkeiten stellt. Hier lohnt insbesondere ein Blick auf die Funktion des Schreibunterrichts im 19. Jahrhundert und in der ersten Hälfte des 20. Jahrhunderts: Die damaligen Schreibgeräte waren von einer materiellen Beschaffenheit, die eine Schreib- bzw. Schriftdidaktik nötig machte, die der Körperhaltung und der Feinmotorik besondere Aufmerksamkeit zukommen liess. Es geht uns aber hier weniger um die historische Perspektive an und für sich, sondern vielmehr um die durch die Besonderheit der Schreibwerkzeuge evozierten Konzepte des Schreibenlehrens bzw. den damit implizierten Vorstellungen, was Schreiben als komplexe (Sprach-) Handlung heisst. ${ }^{1}$

Um diese Konzepte besser verorten zu können, gehen wir zunächst auf die heutige Auffassung von Schreiben als Sprachhandeln ein, um dann in einem zweiten Schritt das Schreiben als Handwerk zu erläutern. Unser Augenmerk gilt dabei auch immer der Frage, wie dies didaktisiert wird. Abschliessend plädieren wir für eine stufenübergreifende Förderung, die einen didaktisch reflektierten Umgang mit den Werkzeugen des Schreibens (Blattlage, Stift-, Hand- und Körperhaltung sowie der dafür nötigen Kontrolle des Sichtfelds) beinhaltet und dabei den Zusammenhang zu anderen Schreibprozessen nicht aus dem Auge verliert. $^{2}$

\section{Sprachhandeln}

In der Deutschdidaktik hat sich das Konzept des sogenannten Sprachhandelns weitgehend durchgesetzt (vgl. Eriksson, Lindauer \& Sieber $2008 \mathrm{zu}$ den Schweizer Bildungsstandards). Sprachkompetenzen werden als Bündel von Fähigkeiten verstanden, mit denen Handlungen mithilfe von Sprache vollzogen werden. Obwohl bei dieser Konzeption des Sprachlernens das Handeln im Vordergrund steht, werden die körperlichen Aspekte dieses Handelns didaktisch nicht modelliert: Dabei läge es nahe, auch die Bewegung der Schreibwerkzeuge, der Schreibhand, die dafür nötigen Körperhaltungen etc. als Teil eines umfassenden Sprachhandelns zu begreifen und zu modellieren. Zwar wird insbesondere in der Schreibdidaktik gerne der Begriff des Werkzeugs herangezogen, dabei werden jedoch körperliche Aspekte explizit ausgeblendet: 
Schreiben zu lernen heißt in unserem Verständnis vor allem und in erster Linie, den Umgang mit den Werkzeugen für das Schreiben zu lernen. Die eigentlichen Werkzeuge des Schreibens sind dabei nicht Stifte, Tastaturen oder Schreibprogramme, sondern sie sind text-sprachlicher Art. (Feilke \& Bachmann, 2014, S. 7)

In diesem Beitrag möchten wir Sprachhandeln konzeptionell und begrifflich so verstehen, dass der Werkzeugcharakter in zweifacher Weise zum Tragen kommt. Wir unterscheiden deshalb zwischen Sprachhandlungen im engeren Sinn, die diejenigen motorischen und kognitiven Aktivitäten umfassen, die zur Produktion von Sprache beim Sprechen und Schreiben sowie beim Zuhören und Lesen nötig sind, und Sprachhandlungen im weiteren Sinn, die diejenigen Aktivitäten umfassen, mit denen komplexe Handlungen mithilfe von Sprache im Sinne von Austin (1986) - «How to do Things with Words» - vollzogen werden. Dabei geht es nicht darum, die beiden «Ebenen» gegeneinander auszuspielen oder gar als Gegensatz zu begreifen - das ist auch nicht die Absicht von Feilke und Bachmann (2014) -, sondern sie in ihrem gegenseitigen Bezug zu sehen.

\section{Schreiben als Handwerk}

Von Beginn der Schweizer Volksschulen war ein Aspekt des Schreibunterrichts eng mit Sprachhandlungen im engeren Sinn und mit einer Didaktik der körperlichen (Schreib-)Handlungen verbunden: Der Erwerb der Handschrift - natürlich auch die damit einhergehende Alphabetisierung - und daran anschliessend der zunehmend souveränere Umgang mit Schreibwerkzeugen waren bereits in den ersten Schweizer Lehrplänen Kern des Curriculums. (Schön-)Schreiben bzw. Schreibtechnik wurde als wesentliche Aufgabe von Schule über alle Stufen etabliert und folgte bis in die 1960er-Jahre hinein als selbständiges Fach einer eigenen didaktisch-methodischen Logik; entsprechend war dieses (Hand-)Schreiben auch vom sonstigen Schreib- und Sprachunterricht getrennt. Das zeigt sich bspw. in den Lektionendotationen im Lehrplan für Mädchenschulen der Stadt Zürich 1833 in den Fächern «Deutsche Sprache» (6-9 Wochenstunden (WS)) und "Kalligraphie» (2-4 WS) oder im Lehrplan des Kantons Aargau 1862 in den Fächern «Sprachfach» ("Anschau-, Sprech-, Lese-, Schreib- und Gedächtnisübungen») (8-10 WS) und «Schreiben» (2 WS). Es zeigt sich zudem in der Konzeption eines Faches «Formenlehre», das Hand-«Schreiben», das (Hand-) «Zeichnen» und die manuellen Tätigkeiten der «geometrischen Formenlehre» umfasste (Lehrplan AG, 1862). Im Vordergrund stand dabei formgetreues Kopieren von Buchstaben(-folgen) und von geometrischen Figuren sowie die damit einhergehende Lesbarkeit bzw. Präzision der bildlichen (Re-)Produktion auf Papier. In diesem Fach wurde das Handwerk beim Führen von Schreib- und Zeichenwerkzeugen durch das Vormachen der 
Lehrperson und das Imitieren der Lernenden - wie bei jedem Handwerk - von der einen zur nächsten Generation weitergegeben.

Allein die physische Beschaffenheit der Schreibwerkzeuge jener Zeit erforderte es, dass die Lehrpersonen vor allem die technischen Aspekte der Körperund Handhaltung explizit vermittelten. Das Schreiben mit einer Bandzug-oder Spitz-Feder setzt nicht nur eine bestimmte Motorik der Hand voraus, sondern das Schreiben mit solchen Schreibgeräten gelingt nur dann, wenn auch die ganze Körperhaltung, insbesondere die Lage der Unterarme, der Schultern, aber auch die Kopfhaltung sowie die Lage des Schreibpapiers in den Blick genommen wird (vgl. Abbildung 1): Der ganze Oberkörper muss in Bezug auf das Schreibblatt und den Schreibtisch optimal ausgerichtet sein, sonst verschmiert die Tinte, das Papier wird zerkratzt, die Linienführung wird unregelmässig etc. Schreiben mit einer Feder heisst also eine weitreichende Kontrolle des Körpers und des (Schreib-)Raums.
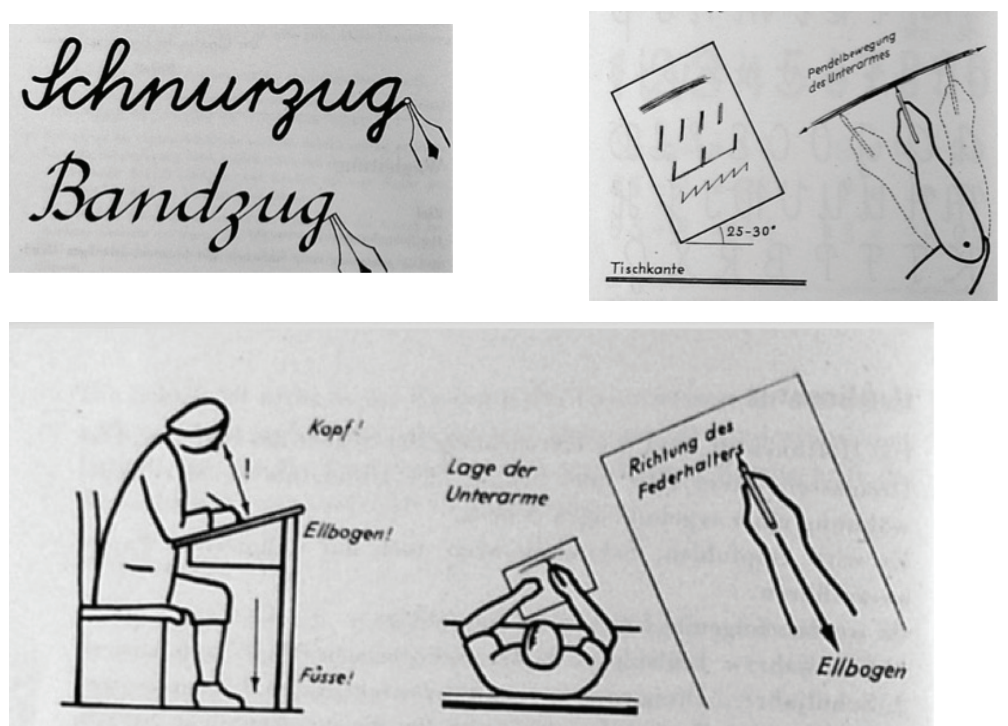

Abbildung 1: "Lehrplan für den Schreibunterricht der deutschen Primar-und Sekundarschulen des Kantons Bern» aus dem Jahr 1946, S. $4 f f$.

Die für diese Schreibgeräte nötige Körpertechnik wurde mit hoher Sorgfalt und bis in die oberen Schulstufen hinein geübt: Unter der Überschrift «Schreiben und Buchhaltung» hält bspw. der Unterrichtsplan für die reformierten deutschen Primarschulen des Kantons Bern (1845, S. 46) als Erstes «Vorübungen» zur «Belehrung über Halten des Körpers, Fassen des Griffels, Lage der Hand» fest.

Der (Schön-)Schreibunterricht wurde also als Handwerk verstanden, als Vermittlung von Techniken zur Bedienung von Werkzeugen, um damit etwas 
zu (re-)produzieren. Die dafür nötige besondere Sorgfalt bei der Vermittlung des Hand-Schreibens und das damit einhergehende Beachten des ganzen «Schreibraums» - das heisst der Sitzhaltung, der Ausrichtung des Oberkörpers, Biegung des Schreibarms, der Stifthaltung, der Lichtverhältnisse, Kontrolle des Sichtfelds usw. - ist in einer Gesellschaft, die noch keine oder kaum Schreibmaschinen verwendet, nötig. Die Koppelung des Schreibunterrichts an berufliche Tätigkeiten zeigt sich sehr deutlich noch in den Berner Lehrplänen des 19. Jahrhunderts: (Schön-)Schreiben und Buchhaltung sind in einem Fach zusammengefasst (Lehrplan BE, 1845, S. 46; Lehrplan BE, 1861, S. 12f.; Lehrplan BE, 1889, S. 11).

Gesellschaftlich und wirtschaftlich war es im Zuge der Industrialisierung und mit dem Wandel zu einer Dienstleistungsgesellschaft von herausragendem Interesse, dass entsprechende Schreib-Handwerker ausgebildet wurden, die die wirtschaftlich relevanten Schriftstücke gut lesbar erstellen konnten: Das betraf nicht nur die Schreibgehilfen in den Ämtern, in den Büros der Fabriken, sondern auch Kleingewerbler, Handwerker etc., welche ihre Korrespondenzen, Verträge etc. selbstständig erledigen und auch die Buchhaltung selbst führen mussten. Entsprechend war es auch nicht wie heute das Ziel, eine persönliche Handschrift auszubilden, vielmehr musste eine für alle lesbare Normschrift und den dafür nötigen Umgang mit nicht leicht handzuhabenden Schreibwerkzeugen vermittelt werden.

Auf dem Hintergrund der oben erwähnten technischen Bedingungen des Schreibwerkzeugs wird nachvollziehbar, dass auch noch auf der Sekundarschule die motorischen Aspekte des Schreibens einen wesentlichen Bestandteil des Schreibunterrichts ausmachten: Es ging beim Handschreiben nicht nur um den primären Schrifterwerb, sondern um ein gezieltes Lernen von Schriftfertigkeiten. Entsprechend war denn auch noch in der ersten Hälfte des 20. Jahrhunderts trotz der zunehmenden Verbreitung von Schreib- und Registriermaschinen ${ }^{3}-$ das Schreiben als Handwerk bis in die Sekundarstufe zu vermitteln. Die hohe Bedeutung des Handschreibens bis ins 20. Jahrhundert hinein und die dafür nötige Vermittlung einer Schreibhaltung, die den ganzen Körper in den Blick nimmt, wird denn auch im 32-seitigen «Lehrplan für den Schreibunterricht der deutschen Primar- und Sekundarschulen des Kantons Bern» aus dem Jahr 1946 augenfällig (vgl. Abbildung 1).

\section{Schreiben als ökonomische Schriftgestaltung}

Während bis Mitte des 20. Jahrhunderts die motorische Seite des Schreibens nicht auf die (Fein-)Motorik der Schreibhand beschränkt wird, sondern die ganze Körperhaltung Gegenstand des Unterrichts bzw. der schreibdidaktischen Modellierung wird, gerät gegen Ende des 20. Jahrhundert zunehmend die Frage nach einer ökonomischen Handschrift, einer Ausgangsschrift als Basis 
für eine persönliche Handschrift und damit einhergehend die Frage nach der Schrifttype in den Fokus. Es entsteht eine Diskussion, die Nottbusch (2008, S. 42) zufolge bereits in den 1960er-Jahren einsetzte und sich zunehmend auf die Schrifttype und die dafür erforderlichen feinmotorischen Aspekte konzentrierte. Im neuen Deutschschweizer Lehrplan 21 wird denn auch der Fokus auf eine persönliche, leserliche und geläufige Handschrift ${ }^{4}$ gelegt: Mit der Automatisierung solch hierarchieniedriger Prozesse wird das Arbeitsgedächtnis entlastet, sodass für hierarchiehöhere Prozesse wie Planen, Formulieren und Überarbeiten mehr kognitive Ressourcen zur Verfügung stehen. Die Körperhaltung wird im Lehrplan $21 \mathrm{im}$ ersten Zyklus noch explizit genannt («können eine günstige Körperhaltung beim Zeichnen und Schreiben einnehmen»), in den Zyklen 2 und 3 wird jedoch nur noch die Feinmotorik expliziert, da die in einem vorhergehenden Zyklus aufgeführten Kompetenzen auch für die folgenden Zyklen gelten sollen.

Diese Verschiebung von einer formgetreuen zu einer persönlichen Handschrift und die damit einhergehende Veränderung in der Konzeption des Schreibunterrichts zeigt sich auch im Handbuch "Didaktik der deutschen Sprache», das ein eigenes Kapitel «Schrift» enthält und sieben Artikel vereint: Es beginnt mit einer Geschichte der «Didaktik des Handschreibens» (Schorch, 2003a), der ein Beitrag zur «Entwicklung des Handschreibens» (Schorch, 2003b) folgt. Die Abbildungen in diesen beiden Grundlagentexten weisen dabei schon deutlich auf die Fokussierung der Schriftgestaltung bzw. Schrifttype. Aufschlussreich sind hier dann auch die weiteren Beiträge zu den «Schulschriften» (Hasert, 2003a) und vor allem auch das Kapitel «Schreibgeräte und Schreiben» (Hasert 2003b), das lediglich Abbildungen der Schreibhand und verschiedener Schreibfedern enthält.

Dass je nach Schreibgerät die ganze Schreib- bzw. Körperhaltung, das Positionieren des Papiers und die Handhaltung als Ganzes schreibdidaktisch modelliert und methodisch ausgestaltet werden müssten - und zwar nicht nur für den Anfangsunterricht -, spielt allenfalls am Rande eine Rolle.

Eine interessante Ausnahme bilden Publikationen zum Schreiben von Linkshändigen (vgl. bspw. Sattler, 2003, Abb. 1, S. 329 oder Marquart \& Sattler, 2010). Hier gerät angesichts spezifischer Probleme, die durch die Linkshändigkeit fürs (Auf-)Schreiben bzw. Transkribieren entstehen, der ganze Körper in den Blick:

Diese [Körperhaltung] ist oft eine Ausweichhaltung, um die Tinte nicht zu verwischen. Hier besteht allerdings ein Instruktionsdefizit. (Sattler, 2003, S. 329)

Auch in neueren Lehrmitteln zur Ausbildung einer persönlichen Handschrift wie Jurt Betschart, Hurschler Lichtsteiner und Henseler Lüthi (2013) wird nicht nur einer sorgfältigen Stiftführung Beachtung geschenkt, sondern ebenfalls die ganze Körperhaltung in den Blick genommen. ${ }^{5}$ Auf den ersten Blick geht es dabei «nur» um «Gesunde Körperhaltung» - so die Kapitelüberschrift -, ein genauerer 
Blick in die Materialien zeigt jedoch: Es geht bei der Reflexion über die Körperhaltung nicht primär um Ergonomie, sondern die Auseinandersetzung mit der Abhängigkeit der Stift- von der Körperhaltung und der Blattlage dient vor allem dem Ermöglichen eines flüssigen Schreibens.

Das hat auch mit der Frage nach der «optimalen» Schreibschrift zu tun, da der Erwerb einer geläufigen und lesbaren Handschrift nicht zuletzt auch von der Schrifttype abhängig ist. Die Diskussion zur Wahl der Schrifttype, wie sie hauptsächlich in den 1980er-Jahren geführt wurde, wird von Nottbusch (2008, S. 43) «als relativ fruchtlos» bewertet, da die Druck- und die verbundene lateinische Ausgangsschrift, die in erster Linie zur Debatte standen, gerade «nicht konsequent auf die Optimierung der Bewegungsabläufe ausgerichtet» sind.

In den letzten Jahren geriet die Frage, welche Schrift die Schüler und Schülerinnen als Erstes lernen sollen, wieder vermehrt in den Fokus. So untersuchten Hurschler Lichtsteiner, Saxer Geiger und Wicki (2010), ob sich 4.-KlässlerInnen in Abhängigkeit vom vermittelten Schrifttyp in den Schreibleistungen unterscheiden, und zwar in Bezug auf Leserlichkeit und Geläufigkeit der Handschrift. Die beiden untersuchten Schrifttypen waren die sogenannte Luzerner Basisschrift - eine teilverbundene Schrift - sowie die ganz verbundene Schweizer Schulschrift, die sog. «Schnürlischrift». In dieser Studie zeigte sich bei beiden Kennwerten ein deutlicher Vorteil zugunsten der Basisschrift (Wicki, Hurschler Lichtsteiner, Saxer Geiger \& Müller, 2014). Bemerkenswert ist zudem, dass vor allem die Jungen hinsichtlich Leserlichkeit von der Basisschrift profitieren konnten. Die Autoren und Autorinnen dieser Studie betonen jedoch, dass der Schrifttyp allein nicht entscheidend sei, dass vielmehr die Art des Schriftunterrichts einen wichtigen Anteil dazu beitragen dürfte, wer wie viel davon profitiert.

Auf Basis dieser Studie und im Hinblick auf den Lehrplan 21 empfiehlt die Arbeitsgruppe Schrift der Erziehungsdirektoren-Konferenz (EDK), auf eine teilverbundene Schrift wie die Basisschrift umzustellen (D-EDK Arbeitsgruppe Schrift, 2013), wobei diese Empfehlung nur für die deutschsprachigen Regionen der Schweiz gilt. Damit wurde (zumindest für die deutsche Schweiz) eine Grundlage geschaffen, sich aus schreibdidaktischer Perspektive mit Blick auf aktuelle Forschungsergebnisse mit dem Modellieren und Vermitteln solch basaler Schreibfertigkeiten (wieder) vermehrt auseinander zu setzen. In diese Richtung zielen denn auch die Kompetenzbeschreibungen im Lehrplan 21 unter der Dimension «Grundfertigkeiten» und die explizite Nennung der Körperhaltung.

In der neueren und vor allem auch empirisch ausgerichteten Schreibforschung zeigt sich zudem, dass der Erwerb der basalen Schreibfertigkeiten, der die «Körperlichkeit» der Textproduktion einschliesst, eine wichtige Voraussetzung darstellt, um erweiterte Schreibkompetenzen in einem ausreichenden Mass ausbilden zu können (vgl. bspw. Schneider et al., 2013). Die enge Verbindung von Formulieren und Transkribieren, wie wir dies zu Beginn kurz ausgeführt haben, spielt dabei eine zentrale Rolle. 


\section{Flüssiges Schreiben als Freisetzung kognitiver Ressourcen}

Sind die Bewegungsabläufe beim Schreiben optimiert, haben die Schüler und Schülerinnen eine flüssige bzw. geläufige Handschrift ausgebildet, reduziert dies die Belastung des Arbeitsgedächtnisses, ${ }^{6}$ sodass kognitive Ressourcen für hierarchiehöhere Aktivitäten wie Ideengenerierung, Formulieren oder Überarbeiten freigesetzt werden. ${ }^{7}$ Dieser Zusammenhang von motorischen und kognitiven Aktivitäten wird mit dem Begriff der Schreibflüssigkeit insofern genauer gefasst, als damit nicht nur eine flüssige Handschrift gemeint ist, sondern auch die Textmenge, die innerhalb einer bestimmten Zeit leserlich, aber auch orthografisch und grammatisch korrekt sowie semantisch passend verfasst wird (vgl. bspw. Dockrell, Connelly, Walter \& Critten, 2012; Malecki \& Jewell, 2003).

Verschiedene Studien weisen darauf hin, dass der Entwicklungsverlauf weder im Hinblick auf eine flüssige Handschrift noch im Hinblick auf Schreibflüssigkeit im erwähnten Sinne nach zwei bis drei Schuljahren abgeschlossen ist (vgl. bspw. Graham, Berninger, Weintraub \& Schafer, 1998; Malecki \& Jewell, 2003). Dennoch werden basale Schreibfertigkeiten über den Anfangsunterricht hinaus von Lehrpersonen eher vernachlässigt, wie Limpo und Alves (2013) oder Medwell und Wray (2007) festhalten.

Im Allgemeinen kann zwar gezeigt werden, dass Transkription bzw. Schreibflüssigkeit bei älteren Schülern und Schülerinnen nicht mehr mit Textqualität korreliert. Es lässt sich jedoch ein indirekter Effekt auf Textqualität via Planungsaktivitäten nachweisen (Limpo \& Alves, 2013): Schüler und Schülernnen der Klassen 7-9 zeigen in dieser Studie bessere Planungsfähigkeiten, wenn sie handschriftlich flüssig schreiben können. Planungsfähigkeiten wiederum korrelieren mit der Textqualität. Zusätzlich können Limpo und Alves (2013) einen indirekten Effekt via Selbstwirksamkeit nachweisen. Das heisst, dass basale Fertigkeiten, insbesondere auch handschriftliche Fertigkeiten, die eigene Wahrnehmung von Schreibkompetenzen beeinflussen und damit auch die Anstrengung, die Persistenz, die Wahl der Aufgabe oder emotionale Reaktionen. Bedenkt man zusätzlich, dass ein systematisches HandschriftTraining bei Sekundarschülern und -schülerinnen mit Schreibschwierigkeiten einen positiven Effekt auf die Textqualität ausüben kann (Christensen, 2005), muss mit Limpo und Alves (2013) betont werden, dass basale Schreibfertigkeiten so lange vermittelt werden sollten, bis alle Schüler und Schülerinnen eine ausreichende Schreibflüssigkeit ausgebildet haben. ${ }^{8}$

Im Verlauf der Schreibentwicklung kommt nicht nur der Transkription, sondern insbesondere auch dem selbstregulierten Schreiben (Graham \& Harris, 2009) sowie der Herausbildung eines Schreibbegriffs, der über die "Oberflächenmerkmale» von Schrift hinausgeht (Schneider, 2011), eine bedeutende Rolle zu. Das Zusammenspiel von Transkription und hierarchiehöheren Aktivitäten - sei es, dass Transkription zusammen mit Formulieren eine Art Flaschenhals 
darstellt, sei es, dass Transkription einen indirekten Effekt auf Planungsaktivitäten ausübt - hat jedoch noch keine didaktisch-konzeptionelle Ausgestaltung erfahren, sodass Schreibhandlungen im engeren und im weiteren Sinn nach wie vor getrennt vermittelt werden. Insbesondere gilt es darüber hinaus empirisch aufzuzeigen, welche Wirkungen mit einer Förderung erzielt werden können, die beide «Ebenen» verbindet.

\section{Schriftdidaktik als Teil einer integrativen Schreibdidaktik}

Die materielle und damit auch körperliche Bedingtheit des Schreibens tritt zunehmend in den Blick der Schreibforschung und Schreibdidaktik. Dabei geht es im Gegensatz zum 19. und 20. Jahrhundert nicht darum, dass den Schülern und Schülerinnen die optimale Körperhaltung fürs Schreiben beigebracht werden soll. Im Gegenteil: Alle bisher vorliegenden Untersuchungen deuten darauf hin, dass es nicht die optimale Haltung zum Schreiben gibt. Eine optimierte Motorik kann sich dann ausbilden, wenn beim Schreibenlernen immer wieder bewusst nach individuellen und effizienten Lösungen der Schreibhaltung gesucht wird. Und dies bis in die höheren Klassen hinein, da sich dort zunehmend eine persönliche Handschrift entwickelt und die Handschrift sich entsprechend auch verändert:

Heutige Modelle beschreiben das motorische Lernen deshalb nicht als die häufige Wiederholung der Lösung für eine bestimmte motorische Aufgabe, sondern vielmehr als die wiederholte Suche nach einer individuellen Lösung für diese Aufgabe. (Marquardt \& Sattler, 2010, S. 1 [Hervorhebungen im Original])

Didaktisch bzw. methodisch heisst das, dass die Lehrpersonen immer wieder die Schüler und Schülerinnen dazu anhalten, ihre Schreib- bzw. Körperhaltung bewusst wahrzunehmen, sie gezielt zu verändern, damit sie so erfahren und reflektieren können, welche Stift- und Körperhaltung ihr Schreibflüssigkeit positiv beeinflusst. Zwar schlägt Schorch (2003b) für höhere Jahrgangsstufen ein tägliches 10-Minuten-Training über einen begrenzten Zeitraum hinweg vor: Entsprechende Hilfsmittel für die Lehrpersonen müssen jedoch als Desiderat bezeichnet werden. ${ }^{9}$ Des Weiteren spielt die Art der Instruktion eine zentrale Rolle: Blosse Abschreibübungen oder ein zu starker Fokus auf formgetreue Schrift zeigen keine oder nur eine geringe Wirkung, während die explizite Vermittlung, die bspw. den Bewegungsablauf visualisiert und verbalisiert oder die Reflexion übers handschriftliche Schreiben einschliesst, grössere Effekte erzielt (Graham et al., 1998). Auf diesem Hintergrund erscheint dieses Desiderat als umso dringlicher.

Eine reflektierte Körperlichkeit im Schreibunterricht bietet darüber hinaus weiteres erst noch zu nutzendes Lernpotenzial, wie nicht nur die bereits erwähnte 
Studie von Limpo und Alves (2013), sondern weitere neuere Forschungen bspw. von Mueller und Oppenheimer (2014) nahe legen: So zeigen Studierende, die zu einer Vorlesung handschriftliche Notizen anfertigten, höheres konzeptuelles Wissen als Studierende, die Vorlesungsnotizen mit einem Laptop erstellten. Mueller und Oppenheimer (2014) interpretieren dies so, dass handschriftliches Notieren mit einer stärkeren kognitiven Verarbeitung einhergeht.

Eine integrative Schreibdidaktik bettet nach Fix (2008) das Schreibenlernen in sinnvolle Zusammenhänge ein und kann in diesem Sinne als Weiterentwicklung einer prozessorientierten Schreibdidaktik gesehen werden. Hier eröffnet sich ein weiteres Feld der sprachdidaktischen Forschung, der didaktisch-konzeptionellen Modellbildung und für methodisch-praktische Entwicklungsarbeiten.

\section{Anmerkungen}

1 Um in der Schreibdidaktik die Materialität bzw. Körperlichkeit des Schreibens in den Blick nehmen zu können, genügt aus unserer Sicht vorerst das Beispiel des «traditionellen» Handschreibens. Zum einen nimmt das handschriftliche Schreiben nach wie vor auch bei Erwachsenen viel Raum ein (Cohen, White \& Cohen, 2011), zum anderen stellt das Tastaturschreiben und insbesondere das Schreiben mit Tablets noch eine jüngere Entwicklung dar. Inwiefern digitales Schreiben die Körperlichkeit des Schreibens, aber auch den Textproduktionsprozess selbst verändert, wäre eine weitere Frage - Analoges gilt für das Schreiben mit Speech-to-Text-Tools-, die bspw. auch Zepter (2014) aufwirft, ohne sie aber zu beantworten.

Die historischen Ausführungen beruhen zu einem wesentlichen Teil auf ersten Ergebnissen aus einem Teilprojekt des SNF-Sinergia-Projekts "Transformation schulischen Wissens seit 1830». Das Teilprojekt «Deutsch und Französisch in der Volksschule der deutschsprachigen Schweiz» wird von Thomas Lindauer mitgeleitet. Das Gesamtprojekt wird von Lucien Criblez, Universität Zürich, geleitet.

2 Zu einem didaktisch reflektierten Umgang mit digitalen Werkzeugen vgl. u.a. Grabowski, Blabusch \& Lorenz (2007) oder MacArthur (2008).

3 Die handschriftliche Buchführung war bis in die 1960er-Jahre in kleineren und mittelständischen Betrieben noch weit verbreitet.

4 Zusätzlich wird das Tastaturschreiben genannt, andere Eingabegeräte wie Tablets jedoch nicht.

5 Vgl. auch Sassoon \& Briem (2010), die solchen Aspekten unter dem Titel «Practical matters» ein ganzes Kapitel widmen. Sie weisen nicht zuletzt auch darauf hin, dass bspw. die Art und Weise, wie der Schreibstift gehalten wird, einem historischen Wandel unterworfen ist.

$6 \mathrm{Zu}$ einer Übersicht über verschiedene Modelle des Arbeitsgedächtnisses, der damit angesetzten Komponenten und ihre Rolle im Textproduktionsprozess vgl. Olive (2012).

7 Das gilt ganz ähnlich auch fürs Tastaturschreiben, vgl. MacArthur (2008).

8 So zeigt auch die Studie von Sturm (2014), dass selbst ein Teil der Berufsschüler und -schülerinnen über eine (zu) geringe Schreibflüssigkeit verfügt.

9 Für die Schweiz wünschbar wäre eine Fortsetzung des Lehrmittels von Jurt Betschart et al. (2013) sowohl für die Mittel- als auch für die Sekundarstufe I. 


\section{Literaturverzeichnis}

Abraham, U. \& Kupfer-Schreiner, C. (Hrsg.). (2007). Schreibaufgaben. Berlin: Cornelsen.

Austin, J. L. (1986). Zur Theorie der Sprechakte (How to do things with words) (E. von Savigny, Trans.). Stuttgart: Reclam.

Becker-Mrotzek, M. \& Böttcher, I. (Hrsg.). (2012). Schreibkompetenz entwickeln und beurteilen (4., überarbeitete Auflage.). Berlin: Cornelsen Scriptor.

Bourdin, B. \& Fayol, M. (2002). Even in adults, written production is still more costly than oral production. International Journal of Psychology, 37(4), 219-227.

Christensen, C. A. (2005). The role of orthographic-motor integration in the production of creative and well-structured written text for students in secondary school. Educational Psychology, 25 (5), 441-453.

Cohen, D. J., White, S. \& Cohen, S. B. (2011). A time use diary study of adult everyday writing behavior. Written Communication, 28(1), 3-33.

D-EDK Arbeitsgruppe Schrift. (2013). Entscheidungsgrundlagen zur Zukunft der Schweizer Schulschrift. Luzern: D-EDK.

Dockrell, J. E., Connelly, V., Walter, K. \& Critten, S. (2012). CBM-W (UK): Curriculum based measures of writing. Administration and scoring manual. Manuskript, London/ Oxford/Coventry.

Eriksson, B., Lindauer, T. \& Sieber, P. (2008). HarmoS Schulsprache - Kompetenzbeschreibungen und Basisstandards. Beiträge zur Lehrerinnen- und Lehrerbildung (BzL), 3, 338-349.

Feilke, H. (2011). Literalität und literale Kompetenz: Kultur, Handlung, Struktur. Leseforum, 1, 1-18. Zugriff am 11.11.2015 unter http://www.leseforum.ch/ fokusartikel2_2011_1.cfm

Feilke, H. \& Bachmann, T. (2014). Werkzeuge des Schreibens - Zur Einleitung. In T. Bachmann \& H. Feilke (Hrsg.), Werkzenge des Schreibens (S. 7-10). Stuttgart: Fillibach bei Klett.

Fix, M. (2008). Texte schreiben: Schreibprozesse im Deutschunterricht. StandardWissen Lehramt (2. Auflage). Stuttgart: UTB.

Grabowski, J., Blabusch, C. \& Lorenz, T. (2007). Welche Schreibkompetenz? Handschrift und Tastatur in der Hauptschule. In M. Becker-Mrotzek \& K. Schindler (Hrsg.), Texte schreiben (S. 41-62). Köln: Gilles \& Francke Verlag.

Graham, S., Berninger, V., Weintraub, N. \& Schafer, W. (1998). Development of handwriting speed and legibility in grades 1-9. The Journal of Educational Research, 92(1), 42.

Graham, S. \& Harris, K. R. (2009). Almost 30 years of writing research: making sense of it all with „the wrath of khan». Learning Disabilities Research \& Practice, 24(2), 58-68.

Hasert, J. W. (2003a). Schulschriften. In U. Bredel, H. Günther, P. Klotz, J. Ossner \& G. Siebert-Ott (Hrsg.), Didaktik der deutschen Sprache (Bände 1-2, Band 1, S. 307-318). Paderborn/München/Wien/Zürich: Schöningh UTB.

Hasert, J. W. (2003b). Schreibgeräte und Schreiben. In U. Bredel, H. Günther, P. Klotz, J. Ossner \& G. Siebert-Ott (Hrsg.), Didaktik der deutschen Sprache (Bände 1-2, Band 1, S. 319-328). Paderborn/München/Wien/Zürich: Schöningh UTB.

Hayes, J. R. (1996). A new framework for understanding cognition and affect in writing. In C. M. Levy \& S. Ransdell (Hrsg.), The Science of Writing: Theories, Methods, Individual Differences and Applications (pp. 1-27). New York: L. Erlbaum Associates.

Hayes, J. R. (2012). Evidence from language bursts, revision, and transcription for translation and its relation to other writing processes. In M. Fayol, D. Alamargot \& V. W. Berninger (Hrsg.), Translation of Thought to Written Text While Composing (pp. 15-25). New York/ London: Psychology Press.

Hayes, J. R. \& Flower, L. (1980). Identifying the organization of writing processes. In L. W. Gregg \& E. R. Steinberg (Hrsg.), Cognitive processes in writing (pp. 3-30). Hillsdale: Erlbaum. 
Hurschler Lichtsteiner, S., Saxer Geiger, A. \& Wicki, W. (2010). Schreibmotorische Leistungen im Primarschulalter in Abhängigkeit vom unterrichteten Schrifttyp (Forschungsbericht Nr. 24). Luzern: Pädagogische Hochschule Zentralschweiz.

Jurt Betschart, J., Hurschler Lichtsteiner, S. \& Henseler Lüthi, L. (2013). Unterwegs zur persönlichen Handschrift (2. Auflage). Luzern: Kantonaler Lehrmittelverlag Luzern.

Limpo, T. \& Alves, R. A. (2013). Modeling writing development: contribution of transcription and self-regulation to portuguese students' text generation quality. Journal of Educational Psychology, 105(2), 401-413.

MacArthur, C. A. (2008). The effects of new technologies on writing and writing processes. In C. A. MacArthur, S. Graham \& J. Fitzgerald (Hrsg.), Handbook of Writing Reserach (pp. 248-262). New York/London: Guilford Press.

Malecki, C. K. \& Jewell, J. (2003). Developmental, gender, and practical considerations in scoring curriculum-based measurement writing probes. Psychology in the Schools, 40(4), 379-90.

Marquart, C. \& Sattler, J. B. (2010). Motorische Schreibleistung von linkshändigen und rechtshändigen Kindern in der 1. bis 4. Grundschulklasse. Et Reha, 49 (1\&2), Sonderdruck, 1-12.

Medwell, J. \& Wray, D. (2007). Handwriting: what do we know and what do we need to know? Literacy, 41(1), 10-15.

Mueller, P. A. \& Oppenheimer, D. M. (2014). The pen is mightier than the keyboard advantages of longhand over laptop note taking. Psychological Science, 25(6), 1159-1168.

Nottbusch, G. (2008). Handschriftliche Sprachproduktion: Sprachstrukturelle und ontogenetische Aspekte (1. Auflage). Niemeyer, Tübingen.

Olive, T. (2012). Working memory in writing. In V. W. Berninger (Hrsg.), Past, present, and future contributions of cognitive writing research to cognitive psychology (pp. 485-503). New York: Psychology Press.

Prior, P. (2008). A sociocultural theory of writing. In C. A. MacArthur, S. Graham \& J. Fitzgerald (Hrsg.), Handbook of writing reserach (pp. 54-66). New York/London: Guilford Press.

Sassoon, R. \& Briem, G. S. (2010). Improve your handwriting. London: Hodder \& Stoughton.

Sattler, J. B. (2003b).Linkshändigkeit. In U. Bredel, H. Günther, P. Klotz, J. Ossner \& G. Siebert-Ott (Hrsg.), Didaktik der deutschen Sprache (Bände 1-2, Band 1, S. 329-340). Paderborn/München/Wien/Zürich: Schöningh UTB.

Schneider, H. (Hrsg.). (2011). Wenn Schriftaneignung (trotzdem) gelingt: Literale Sozialisation und Sinnerfahrung. [Ort]: Juventa.

Schneider, H., Becker-Mrotzek, M., Sturm, A., Jambor-Fahlen, S., Neugebauer, U., Efing, C. et al. (2013). Expertise zur Wirksamkeit von Sprachförderung. Aarau/Köln: Pädagogische Hochschule FHNW, Universität zu Köln, Mercator Institut.

Schorch, G. (2003a). Geschichte der Didaktik des Handschreibens. In U. Bredel, H. Günther, P. Klotz, J. Ossner \& G. Siebert-Ott (Hrsg.), Didaktik der deutschen Sprache (Bände 1-2, Band 1, S. 273-285). Paderborn/München/Wien/Zürich: Schöningh UTB.

Schorch, G. (2003b). Entwicklung des Handschreibens. In U. Bredel, H. Günther, P. Klotz, J. Ossner \& G. Siebert-Ott (Hrsg.), Didaktik der deutschen Sprache (Bände 1-2, Band 1, S. 286-296). Paderborn/München/Wien/Zürich: Schöningh UTB.

Steinmann, M. (2013). Handschriften im Mittelalter. Eine Quellensammlung. Basel: Schwabe. Sturm, A. (2014). Basale Lese- und Schreibfertigkeiten bei BerufsschülerInnen und die Notwendigkeit kompensatorischer Fördermassnahmen. Leseforum, (1), 1-19. Zugriff am 11.11.2015 unter http://www.leseforum.ch/fokusartikel1_2014_1.cfm

Sturm, A. \& Weder, M. (2011). Schreiben als sinnhaftes und sichtbares Tun. In H. Schneider (Hrsg.), Wenn Schriftaneignung (trotzdem) gelingt: Literale Sozialisation und Sinnerfahrung (S. 18-37). Weinheim/München: Juventa. 
Wicki, W., Hurschler Lichtsteiner, S., Saxer Geiger, A. \& Müller, M. (2014). Handwriting fluency in children. Swiss Journal of Psychology, 73(2), 87-96.

Wrobel, A. (2010). Raffael ohne Hände? Mediale Bedingungen und Faktoren des Schreibens und Schreibenlernens (KöBeS). In T. Pohl \& T. Steinhoff (Hrsg.), Textformen als Lernformen (Band 7, S. 27-46). Köln: Gilles \& Francke Verlag.

Zepter, A. L. (2014). Zur Körperlichkeit der Schreibhandlung. Osnabrücker Beiträge zur Sprachtheorie, (85), 151-168.

Schlagworte: Schreibforschung, basale Schreibkompetenzen, Handschreiben, historisch-didaktische Forschung

\section{Écrire: convoquer ensemble cognition et corporalité}

\section{Résumé}

Dans le monde germanophone, l'approche conceptuelle de l'écrit joue un rôle central dans la recherche et les prescriptions en didactique de l'écriture. Aussi pertinente que soit cette approche, elle exclut cependant les conditions matérielles et motrices du processus d'écriture. Les dernières recherches empiriques dans ce domaine montrent que l'acquisition de certaines capacités de base, incluant les dimensions motrices et corporelles, constitue une condition préalable importante à un élargissement des compétences en production écrite. Cet article définit ce que l'on entend par "capacités de base pour écrire», respectivement en "production écrite», et montre le rôle de ces capacités dans l'enseignement et l'apprentissage de l'écriture. Se référant au projet FNS «Transformation des savoirs scolaires depuis 1830", cet article montre aussi l'impact de différents outils d'écriture à travers le temps sur la posture corporelle et les manières d'enseigner l'écriture.

Mots-clés: Recherche sur le processus d'écriture, compétences fondamentales en écriture, écriture manuscrite, recherche historico-didactique

\section{Scrivere: convocare insieme cognizione e corporeità}

Riassunto

Nella ricerca sulla scrittura e sulla didattica in ambito germanofono l'approccio concettuale alla scrittura gioca un ruolo centrale. E tanto rilevante questo approccio che spesso la fisicità dello scrivere viene posta in secondo piano. Nelle più recenti ricerche empiriche sulla scrittura emerge che lo sviluppo delle competenze base di scrittura, che riguardano in modo particolare la „corporeità” della produzione testuale, rappresentano dei prerequisiti per uno sviluppo significativo delle competenze avanzate. In questo articolo viene mostrato da un lato, cosa si intenda con i termini "competenze di base di scrittura" e "produzione scritta", 
e quale ruolo giochino per lo sviluppo della scrittura e per la didattica della prima lingua (qui, il tedesco); dall'altro lato viene presentato il significato della materialità (ad esempio, dei diversi tipi di strumento di scrittura) per la didattica del comportamento fisico nella scrittura attraverso i cambiamenti storici, in relazione al progetto FNS «Transformation schulischen Wissens seit 1830».

Parole chiave: Ricerche sul processo di scrittura, competenze base legate alla scrittura, calligrafia, ricerca storico-didattica

\section{Writing: Combining Cognition and Corporeality}

\section{Summary}

The notion of conceptual literacy plays an important role in writing research and writing instruction within German-speaking countries. However, the material and motor components of writing are often neglected in this framework. Recent empirical research show that the acquisition of basic transcription skills, including physical and corporeal dimensions, is an important pre-condition to successful advanced writing skills learning. This article defines the concept of basic transcription skills and their role in the acquisition of writing production skills, as well as their importance for writing instruction. With respect to the SNF-project «Transformation schulischen Wissens seit 1830» (Transformation of school knowledge since 1830), the impact of materiality - i.e. the influence of the different types of writing instruments used throughout the years - on writing, posture and writing instruction are examined.

Keyords: Writing research, basic writing skills, handwriting, history of teaching 
